Villa Sánchez, A. (2019). Liderazgo: una clave para la innovación y el cambio educativo. Revista de Investigación Educativa, 37(2), 301-326.

DOI: http://dx.doi.org/10.6018/rie.37.2.365461

\title{
Liderazgo: una clave para la innovación y el cambio educativo
}

\section{Leadership: a key to innovation and educational change}

\author{
Aurelio Villa Sánchez \\ * Catedrático Emérito de la Universidad de Deusto
}

\begin{abstract}
Resumen
Este artículo constituye una revisión temática desde el punto de vista experiencial del impacto del liderazgo en el ámbito educativo y muy especialmente en los procesos de innovación y en el campo educativo. Se aportan datos de estudios y experiencias con diferentes modelos de liderazgo, llevados a cabo durante esas cuatro últimas décadas que nos permiten extraer algunas consideraciones y conclusiones. Una perspectiva del liderazgo que está emergiendo con fuerza y que actualiza el denominado liderazgo pedagógico resaltado por el movimiento de las escuelas eficaces y el de la mejora.

Se recogen estudios empíricos en los que se han aplicado diversos modelos de liderazgo. En primer lugar, el modelo de liderazgo situacional de Fiedler, muy utilizado en la década de los 70 y que hemos aplicado en un contexto de reforma de las enseñanzas medias. Un segundo modelo examinado es el Liderazgo Transformacional de Bernard Bass llevado a cabo en dos contextos distintos: en el ámbito empresarial y en el ámbito educativo. Un tercer enfoque es el propuesto por Kenneth Leithwood y colaboradores de lo que se ha denominado Prácticas eficaces de liderazgo en el ámbito educativo, modelo que se ha validado en diversos países. Finalmente, se presenta una exploración del liderazgo emocional, tema del que viene enfatizándose la importancia de la inteligencia emocional como una de las variables que mejor explican la eficacia y satisfacción del liderazgo educativo.

Palabras clave: Liderazgo situacional; liderazgo transaccional; liderazgo transformacional; liderazgo pedagógico.
\end{abstract}

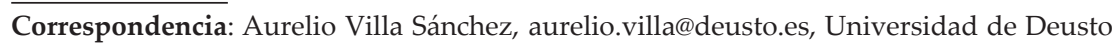




\begin{abstract}
This article constitutes a review under an experiential approach of the leadership impact in education, and especially in the processes of innovation and educational change. It is provided data from studies and experiences with different models of leadership, carried out during these last four decades that allow us to draw some considerations and conclusions. A perspective of leadership that is strongly emerging and that updates the so-called pedagogical leadership highlighted by the movement for effective schools and improvement.

Empirical studies are collected in which various leadership models have been applied. Firstly, Fiedler's situational leadership model, which was widely used in the 1970s and which we have applied in the context of the secondary education reform. A second model examined is Bernard Bass' Transformational Leadership carried out in two different contexts: in business and in education. A third approach was the one proposed by Kenneth Leithwood and collaborators of what has been called Effective Leadership Practices in Education, a model that has been validated in several countries. Finally, an exploration of emotional leadership is presented, an issue that has been emphasizing the importance of emotional intelligence as one of the variables that best explain the effectiveness and satisfaction of educational leadership.

Keywords: situational leadership; transactional leadership; transformational leadership; educational leadership.
\end{abstract}

\title{
Dedicatoria
}

Este artículo representa el trabajo de muchas personas con las que he colaborado y he tenido la suerte de encontrármelas en algún momento de mi vida académica: colegas y estudiantes que me han dado la oportunidad de aprender y disfrutar, a las que manifiesto mi agradecimiento, y muy especialmente a los miembros de mi equipo.

\section{Introducción}

El concepto de liderazgo desde el punto de vista académico-científico nace en la década de los años 40 en las universidades estadounidenses de Ohio y Michigan. El origen de las primeras teorías sobre el liderazgo es de sobra conocido y pueden verse en numerosos tratados, manuales y libros sobre liderazgo (Bass, 1981; Pascual, 1987; Stodgill, 1974).

Como concluye Pascual de su revisión bibliográfica, en la investigación sobre el liderazgo hasta ahora (se refiere hasta el 90), podrían señalarse las siguientes conclusiones:

- El liderazgo no es historia de los "Grandes Hombres".

- El liderazgo no se puede explicar teniendo en cuenta exclusivamente los rasgos o características de la personalidad.

- No existe un estilo ideal de liderazgo válido para todas las situaciones.

- El liderazgo es un fenómeno, es decir, de congruencia entre persona y situación.

- El liderazgo es un fenómeno contingencial, resultado de una relación causa-efecto entre las personas y las variables de la organización. 
En España en el ámbito educativo, el liderazgo comienza a estudiarse a mediados de la década de los años 70, si bien es verdad que con cierta resistencia, por ser un término proveniente de la bibliografía empresarial que no encajaba con los prejuicios existentes en la época. Sin embargo, el liderazgo es un enfoque tan potente que se impuso paulatinamente y en la actualidad es difícil encontrar un estudio de cambio o innovación educativa en el que no se mencione o trate el liderazgo.

\section{La concepción de los directores escolares en España}

Los antecedentes del liderazgo en educación, se encuentran en los estudios de liderazgo empresarial, que paulatinamente penetran en el espacio de la educación. Desde las aportaciones pioneras de las Universidades de Ohio y Michigan, fueron desarrollándose instituciones que, desde el enfoque de la dinámica de grupos y de los estudios de actitudes (Research Center for group Dynamics), van aportando estudios e investigaciones a partir de los estudios de Lewin, Lippit y White (1939).

La ley General de Educación del 70 fue la última ley bajo el régimen franquista que, sin embargo, inició una apertura en la educación creando los Institutos de Ciencias de la Educación que, con sus luces y sus sombras, cambiaron el panorama educativo, aportando contactos e ideas de movimientos europeos, y especialmente anglosajones. En esta década comienza a tomar importancia la formación de los directores, y después de una trayectoria histórica de la dirección como cuerpos de funcionarios del Estado, comienza una época en la que interviene el profesorado en la elección de la dirección. Las experiencias de este sistema fueron objeto de numerosas publicaciones, jornadas y congresos para su análisis y debate. Junto con Portugal, España es el único país en Europa cuya dirección es elegida democráticamente por su profesorado.

Para entender hoy el tema del liderazgo en los centros educativos hay que retrotraerse al sistema de selección de la dirección que se crea en España después de esta Ley. Como señalábamos en un artículo que analizaba las tres últimas décadas del siglo XX sobre la situación de la dirección en España (García-Olalla, Poblete \& Villa, 2006):

La función directiva es un ámbito de especialidad profesional en todos los países europeos excepto en España, que mantiene un sistema de elección democrática del director entre los profesores del centro. Este enfoque ha sido investigado no sólo por investigadores españoles sino que ha interesado a investigadores extranjeros por su singularidad. Los resultados de las investigaciones son bastante nítidos: el sistema no funciona, existen dificultades de encontrar profesores que deseen asumir la función directiva en el contexto y situación actual. En el año 1991, presentamos una visión panorámica de los procedimientos de los directores escolares europeos que contrasta con el sistema de selección español (Pascual \& Villa, 1991, p. 15).

Las diferencias establecidas desde un punto de vista comparativo no sólo se deben a las diversas formas de seleccionar a los directores escolares sino también a las funciones distintas que llevan a cabo. En Inglaterra y Gales la dirección desarrolla un modelo gerencial, ocupándose de gestionar tanto los recursos económicos (tienen su plan eco- 
nómico anual) como los recursos humanos. En Francia, el director es un funcionario representante del estado con un gran poder y autoridad. En los Países Bajos, y los Países Nórdicos, la dirección escolar desarrolla las tareas de gestión y asesoramiento pedagógico, y es la responsable de la calidad del centro ante las autoridades locales.

Los resultados de estudios e investigaciones durante esas tres décadas pueden verse en las actas de los congresos sobre dirección (Pascual \& Villa, 1991; Villa, Auzmendi, \& Villardón, 1996; Villa, 2000, 2004, 2008) además de otros estudios dedicados a esta temática (Estruch, 2002a; Estruch, 2002b; Gairín \& Villa, 1996; García-Olalla, 1998; García Olalla \& Poblete, 2003; Villa \& García-Olalla, 2003).

En la misma época, en Reino Unido se estaban llevando a cabo reformas y cambios trascendentales en los centros educativos. Como indica Bayley (2000, p. 131):

en Inglaterra y Gales, existen 27.000 escuelas estatales, organizadas y controladas en su mayor parte por 117 autoridades de educación elegidas localmente (LEAs), después de la Ley de Reforma de 1988, las escuelas han experimentado grandes cambios tanto en los aspectos internos como externos, que han repercutido en su organización y gestión.

La dificultad de una dirección elegida por los propios profesores del claustro y que en una gran parte de los casos no se deseaba asumir, es que el ejercicio de la dirección se relaja y existe un abandono del liderazgo educativo.

La falta de deseo de pasar a ejercer el cargo de la dirección en los centros públicos ha sido una constante en estas décadas hasta tal punto que la administración se veía obligada a nombrar a un miembro del claustro docente a asumir la dirección y esto sucedía en torno a un $50 \%$ de los casos o incluso más dependiendo del nivel educativo del centro.

Esta debilidad o falta de asunción de muchos directores se nota en las actitudes y comportamientos que muestran y asumen o no asumen ante el uso de los datos de evaluación como parte del sistema de gestión. Y este fue el propósito fundamental de la siguiente investigación, Villa et al. (1996).

Este estudio se basó en 172 centros educativos de las Comunidades Autónomas del País Vasco, Navarra, Canarias y una pequeña participación de Andalucía (este grupo provenía de un seminario de dirección de ocho centros), tal como se refleja en la tabla 1:

Tabla 1

Ubicación y número de participantes del estudio de Villa et al. (1996).

\begin{tabular}{lccc}
\hline Comunidad Autónoma & Provincia & No de centros & oo de participantes \\
\hline País Vasco & Alava & 11 & 44 \\
& Guipúzcoa & 20 & 89 \\
& Bizkaia & 37 & 134 \\
Navarra & Navarra & 40 & 148 \\
Canarias & Tenerife & 19 & 53 \\
& Las Palmas & 37 & 136 \\
Andalucía & Sevilla & 8 & 24 \\
TOTAL & & 172 centros & 628 participantes \\
\hline
\end{tabular}


Esta investigación analizó la actitud y comportamiento de la dirección (miembros de los equipos directivos) ante el uso de la evaluación, medido por los siguientes factores con sus respectivos índices de consistencia interna según el alpha de Cronbach:

- Factor I. Uso de los datos del rendimiento escolar $(\alpha=.94)$ con tres subfactores: Uso de los datos del rendimiento como evaluación formativa $(\alpha=.85)$, para favorecer la eficacia escolar $(\alpha=.90)$, y para concienciar y responsabilizar de la práctica docente $(\alpha=.77)$.

- Factor II. Control de la dirección de objetivos y evaluación $(\alpha=.90)$.

- Factor III. Implicación del profesorado y alumnos en la evaluación $(\alpha=.83)$.

- Factor IV. Énfasis en el aprendizaje $(\alpha=.76)$.

- Factor V. Impacto de las calificaciones (alpha $\alpha$ ha=.58).

Los resultados obtenidos indican, como cabía esperar, que los equipos que tienen más asumida su función de toma de decisiones, muestran unas opiniones más favorables con respecto al uso de la evaluación. Consideran en mayor medida, que el uso de los datos de rendimiento sirve para favorecer la eficacia escolar y para responsabilizarse de la práctica docente, así como para facilitar la evaluación formativa. Es lógico que, si una persona asume el cargo de director/a por imposición administrativa pero que no lo desea, y además piensa que ella es un compañero/a más, le cuesta asumir las funciones que conllevan responsabilidad directa en aspectos como la evaluación en general y más en la evaluación docente. Esta es una de las debilidades de un sistema elegido por el propio profesorado, que cuando llega al cargo de la dirección, les cuesta actuar como verdaderos directores.

\section{Tipos de Liderazgo}

Se pueden encontrar en la literatura un sinfín de términos que adjetivan el liderazgo como: liderazgo servidor (Greenleaf, 1977), liderazgo transformacional, (Bass, 1985), liderazgo visionario (Sashkin, 1998), liderazgo lateral (Fisher \& Sharp, 1999), liderazgo distribuido (Gronn, 2002), liderazgo positivo (Cameron, Dutton \& Quinn, 2003), liderazgo de máximo nivel, (Blanchard, 2007), liderazgo heroico (Lowney, 2004), liderazgo compartido (Álvarez, 2010), liderazgo poliédrico (Castiñeira \& Lozano, 2012), liderazgo peregrino (Segarra, 2018), liderazgo emocional (Goleman, 1996, 1998), solo por citar algunos de los numerosos existentes.

\section{Liderazgo Transaccional y Liderazgo Transformacional}

Bernard Bass dio un paso muy significativo en el enfoque de liderazgo. Después de varios años dedicándose al estudio del liderazgo, y basándose en el marco teórico establecido por James McGregor Burns, quién había creado el concepto de liderazgo transformacional como el liderazgo que es capaz de centrarse en los seguidores para motivarlos y desarrollar sus potencialidades, y avanzar en un tipo de liderazgo que deja atrás un liderazgo de bajo nivel centrado en una transacción entre líder y seguidores, de ahí el nombre de liderazgo transaccional. Hay que reconocer a Bass el mérito de 
lograr que esta teoría fuese llevada a la práctica y poder demostrar científicamente que el liderazgo transformacional obtiene unos resultados significativamente más importantes que el liderazgo transaccional.

En 1985, Bernard Bass diseña el instrumento para evaluar el liderazgo transformacional y a partir de ese momento ha sido aplicado y replicado en multitud de investigaciones en muy diferentes lenguas, diversos contextos (empresas, educación, ámbitos de sociedades no lucrativas, ámbitos religiosos y militares).

El liderazgo transformacional, entendido como el liderazgo que pone el énfasis en el desarrollo de la autoestima de los empleados que se identifican con la misión del líder. Se produce cuando una o más personas se articulan con otras de tal modo que los líderes y los seguidores se eleven unos a otros a niveles superiores de motivación y moralidad. El liderazgo Transaccional es pragmático, requiere un ojo agudo para captar la oportunidad y una mano hábil para el regateo, la persuasión, la reciprocidad (McGregor Burns).

El liderazgo transformacional está compuesto por cuatro grandes factores:

- Carisma (que posteriormente Bass modificó por Influencia Idealizada).

- Inspiración o Motivación Inspiracional.

- Estimulación Intelectual.

- Consideración Individual.

El liderazgo Transaccional está configurado por dos factores:

- Recompensa Contingencial.

- Dirección por Excepción.

El liderazgo cuando no se ejerce se convierte en no liderazgo o "laissez-faire".

Véase en el apartado de estudios empíricos las investigaciones del liderazgo transformacional en el contexto empresarial y en el ámbito educativo.

\section{Liderazgo Emocional y Resonante}

La Inteligencia Emocional (IE) es el factor de éxito más importante en cualquier carrera, más que el Coeficiente Intelectual $(\mathrm{CI})$ o la pericia técnica. Y cuanto más elevada es la posición de alguien en una organización, más importante es la IE; la IE es responsable de entre el 85 y el 90\% del éxito de los líderes de las Organizaciones (Warren Bennis).

Afrontar los grandes cambios requiere, entre otras cosas, de la habilidad de percibir y comprender el impacto emocional del cambio en nosotros mismos y en los demás. (Goleman \& Cherniss, 2013).

En la formación que realizamos con directivos en educación, aplicamos el instrumento de diagnóstico de inteligencia emocional denominado WLEIS, que mide cuatro aspectos clave de la inteligencia emocional: Percepción Intrapersonal (capacidad del individuo para entender sus emociones profundas y ser capaz de expresar estas 
emociones de forma natural), Percepción Interpersonal (capacidad de evaluación y reconocimiento de las emociones en los demás. Esto se relaciona con la capacidad de las personas para percibir y entender las emociones de las personas alrededor de ellos). Asimilación Emocional (capacidad de las personas para regular sus emociones, lo que permitirá una recuperación más rápida de trastornos psicológicos). Regulación Emocional (capacidad de las personas para utilizar sus emociones, dirigiéndolos hacia actividades constructivas y desempeño personal) (Urquijo, Extremera \& Villa, 2015).

Este instrumento ha sido aplicado en muchas investigaciones y consideramos que es un instrumento muy adecuado para ayudar a conocerse a sí mismo, reconociendo los aspectos fuertes y débiles desde la perspectiva emocional, que es algo absolutamente necesario para cualquier líder. Dado que las emociones intervienen en todos nuestros actos y comportamientos, autorreconocerlas es un aspecto muy importante para ejercer el liderazgo.

Goleman, Boyatzis y McKee (2002) definen el liderazgo resonante cómo el liderazgo que sintoniza con los sentimientos de las personas y los encauza en una dirección emocionalmente positiva. Uno de los signos más potentes del líder resonante es el optimismo y entusiasmo que exhiben sus subordinados. El modo en que el líder gestiona y canaliza las emociones para que un determinado grupo alcance sus objetivos depende de su grado de inteligencia emocional. Los líderes emocionalmente inteligentes alientan en sus subordinados cierto grado de bienestar que les llevan a compartir las ideas, aprender los unos de los otros, asumir decisiones grupales y permitir, en suma, que las cosas funcionen.

Goleman (2013) presenta un marco de competencias de liderazgo emocional, como puede verse en la figura 1.

\section{EL MARCO DE COMPETENCIAS}

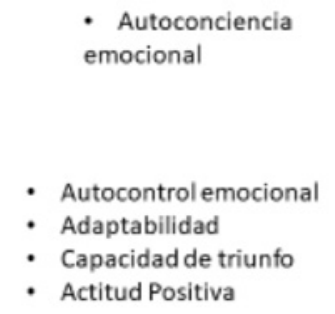

- Autoconciencia emocional

- Autocontrolemocional

- Adaptabilidad

- Capacidad de triunfo

- Actitud Positiva

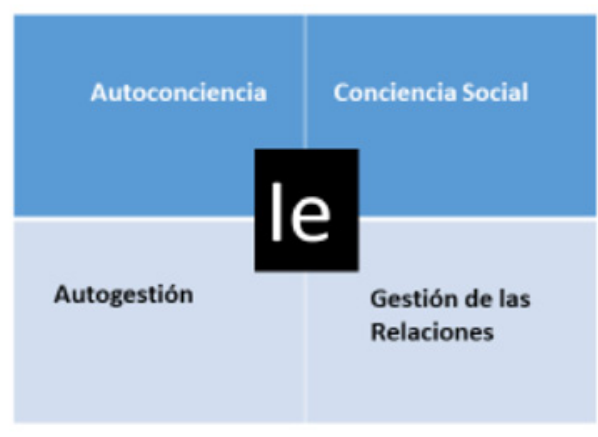

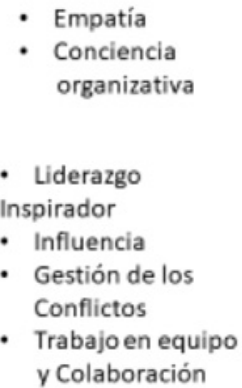

- Empatía

Conciencia organizativa

- Liderazgo

Inspirador

- Influencia

- Gestión de los Conflictos y Colaboración

Figura 1. Liderazgo. El poder de la Inteligencia Emocional (Goleman \& Cherniss , 2013, p. 15).

Boyatzis, McKee y Johnston (2008) en su obra "El líder emocional" después de analizar una serie de mitos sobre el liderazgo, proponen un programa para su desarrollo a base de ejercicios para realizar como parte del entrenamiento para el avance del liderazgo emocional. 


\section{Liderazgo Servidor o Servicial}

Se debe a Greenleaf (1977) el término de líder servidor, que dio lugar a que muchos estudiosos siguieran utilizando este término e intentando operativizarlo en sus investigaciones.

Russell y Stone (2002), parten de la consideración de Greenleaf de "la crisis de liderazgo" basándose en la ineficiencia por parte de los centros, universidades y seminarios que han fracasado en la formación de los estudiantes sobre los roles que necesitarán desarrollar en la Sociedad.

En su extensa revisión de autores que han seguido el enfoque del liderazgo servidor, han recogido nueve características de las investigaciones precedentes:

a) Visión, b) Honestidad, c) Integridad, d) Confianza, e) Servicio, f) Modelo, g) Pionero, h) Apreciación de los demás, y finalmente i) Empoderamiento.

Estos nueve atributos constituyen los fundamentos del modelo de liderazgo servidor. Este enfoque de liderazgo puede potencialmente cambiar las organizaciones y la sociedad porque estimula la metamorfosis personal y organizacional, según estos autores.

Segarra (2018) presenta en su reciente libro un modelo que denomina L.I.D.E.R (que configura las competencias necesarias según el autor). Modelo que podríamos enmarcar en un liderazgo servidor y compartido que pretende empoderar a las personas.

Localizar: construir un propósito común que alinee y dé sentido, que oriente. Inspirar a las personas para que se comprometan con él. Desarrollar los conocimientos y recursos necesarios. Ejecutar mediante una ejecución ambiciosa y disciplinada. Referente: Construir un equipo de líderes referentes y motor de energía positiva. Basado en este modelo, propone un plan o metodología para entrenar el liderazgo.

\section{El liderazgo distribuido}

Zepke (2007) señala que el liderazgo distribuido es un concepto complejo y multifacético. El término fue discutido por primera vez en la literatura de la psicología social en la década de 1950, ganó adeptos a finales de 1990, concretamente dentro de la literatura relacionada con la mejora de la escuela (Spillane, Halverson \& Diamond, 2001; McInermy, 2003).

En la revisión llevada a cabo por Bennet et al. (2003) sobre 30 estudios de liderazgo distributivo descubrió la falta de definiciones claras sobre este término. Dedujo sin embargo, que era lo suficientemente diferente de otros conceptos de liderazgo cómo para tenerlo en cuenta como otro tipo de liderazgo.

Gronn (2002) identificó dos significados distintivos del liderazgo distribuido. En uno, el liderazgo es aditivo, extendido entre múltiples miembros de un grupo. Cada miembro del grupo puede adquirir un rol de liderazgo con el fin de contribuir a la actividad del grupo. En el otro, el liderazgo es ejercido de una manera más holística, "aquí la conducta que compone la unidad de análisis es una acción coordinada más que una conducta agregada" (p.656). El autor sugiere que el significado de acción coordinada del liderazgo distributivo es el más significativo. Distinguió tres patrones: en primer lugar, la colaboración espontánea, personas con diferentes habilidades y atributos interactúan en relaciones productivas para completar una tarea de interés 
común. En el segundo patrón, reparto de roles, dos o más personas trabajan constructivamente dentro del marco implícito de comprensión. El liderazgo cambia a medida que los colaboradores aprenden a reconocer las habilidades y atributos de una de las personas. En el tercer patrón, las relaciones formales en las estructuras acordadas emergen para formalizar el trabajo conjunto, permitiendo que los roles de liderazgo sean reconocidos oficialmente.

En síntesis, el liderazgo distribuido es una tarea de equipo que es conveniente asumirla desde esta perspectiva holística en la que el todo es más que la suma de las partes. Es como un equipo de fútbol, dónde cada jugador desempeña un rol (portero, defensa, medio delantero), pero cada uno se siente parte integrante del equipo, que es más que cada uno de ellos o ellas por importantes que sean, y tienen unas metas comunes y compartidas que es lo que les da la unión, la coherencia y la razón de ser, su identidad. (Villa, 2013, p.339).

\section{El liderazgo para el aprendizaje}

Como señalábamos en otro lugar (Villa, 2013, p. 335), en la década de los 70 y 80 el liderazgo instruccional fue uno de los temas estudiados. Uno de los problemas fundamentales es la falta de una definición clara y unívoca del concepto de liderazgo instruccional. Sheppard (1996) distingue dos conceptos que se pueden encontrar en la bibliografía de esas décadas, y que el autor clasifica estas dos definiciones cómo restringida y amplia. La definición restringida centra el liderazgo instruccional como una entidad independiente de la gestión (Murphy,1998). En esta concepción restringida, el liderazgo instruccional está definido cómo las actividades que están relacionadas directamente con el proceso de enseñanza-aprendizaje, conductas observadas en la supervisión en el aula. En la definición más amplia, el liderazgo instruccional implica todas las actividades de liderazgo que afectan el aprendizaje de los estudiantes. Las conductas de gestión rutinaria se consideran que contribuyen tanto a la mejora de la enseñanza y el aprendizaje cómo para dirigir las conductas instruccionales (Sheppard, 1996, p.326).

Peter Earley y Grean (en prensa, 2019) siguiendo a Simkins (2012) destacan que en Inglaterra, la Ley de Reforma Educativa de 1988 y la introducción de la descentralización y la gestión basada en la escuela han significado que las habilidades y capacidades requeridas de los líderes escolares han tenido que cambiar. Simkins (2012) ofrece tres eras distintas del desarrollo del liderazgo escolar en Inglaterra: "la era de la administración"; "La era de la gestión"; y "la era del liderazgo".

La era de la Administración fue desde 1944 hasta mediados de la década de 1980, cuando los maestros tenían altos grados de autonomía y el trabajo de los administradores escolares, como «profesionales principales», se sustentaba comúnmente en valores derivados de la práctica profesional, situados dentro de un marco burocrático de reglas y procedimientos.

La segunda era de la Gestión, desde mediados de la década de 1980 hasta 1997, se caracterizó por las crecientes preocupaciones de los gobiernos sobre el desempeño de las escuelas. Simkins (2012) señala que los administradores se reconstituyeron en 
la política cómo «gerentes» que fueron responsables de cómo se desempeñaron sus escuelas en comparación con los criterios definidos centralmente.

La tercera era contemporánea de Liderazgo comenzó en 1997, sosteniendo el autor que esta se construyó sobre el marco de gestión de la responsabilidad y la autonomía de la escuela, pero añadió el «liderazgo» como un «impulso de mayor capacidad para instituir el cambio y mejorar el desempeño en el sector público» (Simkins 2012, p. 625).

La investigación realizada en el sector comercial (Pillans, 2015, p. 16), identificó tres tendencias clave que impulsan la forma en que las organizaciones ven el liderazgo y cómo se desarrollan los futuros líderes:

- El liderazgo se ve cada vez más como un proceso. Por ejemplo, Heifetz (1994) define el liderazgo como el proceso de movilización de personas para enfrentar desafíos difíciles

- El liderazgo compartido o distribuido está en aumento. Se considera que el liderazgo es un requisito central para todos los miembros de la organización, independientemente de que sean responsables de administrar a otros o no.

- Existe un creciente deseo por los "líderes auténticos", que sean confiables, genuinos y consistentes. Se considera que un enfoque clave para el desarrollo del liderazgo ayuda a las personas a comprender su propio propósito, valores y carácter, y lo que eso significa para su estilo de liderazgo (Earley \& Grean, 2019).

A medida que las escuelas han ganado más autonomía, más importante es el papel de los líderes escolares, especialmente los directores y, más recientemente, los directores ejecutivos. Las políticas relacionadas con la descentralización y la autonomía escolar han otorgado poderes considerables a los líderes escolares que, como hemos señalado en otros lugares: se sientan en el punto de apoyo de los sistemas de alta rendición de cuentas de alta autonomía y se espera que resuelvan las paradojas políticas tanto de la competencia como de la cooperación. (Earley \& Greany, 2017, p. 4).

Bolívar (2012) destaca que: “La mejora y eficacia escolar, en último extremo, se juega en los procesos de enseñanza aprendizaje que tienen lugar en las aulas. De ahí que el papel de los equipos directivos y su función de liderazgo se deba centrar en crear condiciones para que estos sean más ricos y productivos". (p. 47).

MacBeath (2011) presenta una visión muy crítica del liderazgo escolar:

La escuela es un lugar de pocos líderes y muchos seguidores y la pasión es algo que debe ser dejado en la puerta de entrada. Al cabo de diez o doce años de escolaridad es notable cuán poco nos llevamos de las 15.000 horas de exposición al currículo escolar, pero aprendemos lecciones imborrables sobre relaciones, dualidades y paradojas, disciplina y castigo, impulso y control, obediencia y subversión. (MacBeath, 2011, p. 20).

Este autor parte de una perspectiva muy crítica sobre el efecto escolar, cuando atestigua que cuatro décadas de investigación sobre logros escolares han mostrado de 
manera consistente que los efectos de la escuela son mínimos si se comparan con la influencia de los padres, la familia, el vecindario, aun cuando el liderazgo aparece como un tema recurrente en prácticamente todos los estudios, aunque posiblemente estamos ante una postura excesivamente crítica a juzgar por los datos presentados por diversos estudios, como por ejemplo el meta-análisis de Marzano, Waters y McNulty (2005).

La sugerencia realizada por varios autores, MacBeath, Elmore, Day (citado en Villa, 2013) es tener en cuenta el contexto y llevar a cabo la rendición de cuentas, primeramente de forma interna y posteriormente de manera externa. La rendición de cuentas interna, significa tener transparencia y presentar los resultados obtenidos de la acción directiva e institucional ante la comunidad educativa en, primer lugar, y luego prepararse para rendir cuentas ante la administración y la sociedad, normalmente a través de las agencias de calidad correspondientes o de la inspección educativa.

Para entender esta postura del énfasis del liderazgo para el aprendizaje Mulford (2006) afirma:

este trabajo identifica algunas de las novedades internacionales en dicho contexto en el liderazgo de la educación y en las escuelas. Primero se centra en trabajos que emanan de la OCDE y luego incluye novedades recientes en el Reino Unido y Australia. Todos empiezan en el nivel más general, preguntando sobre qué tipo de educación sirve mejor a la sociedad ahora y en el futuro. (Mulford, 2006, p. 2).

Para Mulford (2006) este cambio de enfoque no resulta nada fácil, y entiende el aprendizaje cómo una interacción, en la cual los aprendices descubren por sí mismos, piensan en lo que han aprendido y cómo. Brevemente, el aprendizaje es co-creado. Pero apoyar este aprendizaje no será fácil... muchas de las piezas básicas de la educación tradicional, la escuela, el grupo, la clase, la lección, la pizarra y el profesor delante de una clase de treinta niños, podrían ser vistos como obstáculos para al aprendizaje personalizado.

El informe de la OCDE (2014) afirma que el liderazgo no se ejerce bajo la forma de una indicación única del camino a seguir, sino como un proceso continuo que incluye las estrategias de la puesta en práctica, así como las visiones directrices. Tres aspectos: visión del aprendizaje, estrategias de cambio, y compartir (responsables, profesores, estudiantes y destinatarios). La naturaleza misma del liderazgo pedagógico implica que debe ser compartido.

Los nuevos entornos pedagógicos llegarán a englobar todos los diferentes aspectos, actividades y relaciones según los siguientes principios:

- Concentrarse sobre el aprendizaje y la inversión personal.

- Asegurar que el aprendizaje es social y a menudo colaborativo.

- Estar perfectamente de acuerdo con la motivaciones y emociones del aprendizaje.

- Ser muy sensibles a las diferencias entre los individuos.

- Recurrir a las evaluaciones conforme a los objetivos pedagógicos, insistiendo fuertemente sobre el feedback formativo.

- Promover la conexión horizontal a través de las actividades, y las materias en el seno de la escuela, pero también fuera. (OCDE, 2014, p. 15). 


\section{Prácticas exitosas de liderazgo educativo}

Quizás se debe a Leithwood y colaboradores la propuesta de prácticas más exitosas basadas en los resultados de la investigación y coincidentes de su puesta en práctica en diferentes países con culturas y sistemas educativos muy distintos. Como describíamos en otro lugar (Villa, 2009), las cuatro prácticas señaladas por Leithwood y colaboradores, contienen cuatro dimensiones:

1. Establece direcciones.

Las prácticas agrupadas en esta dimensión incluyen las acciones de los líderes que se dirigen al desarrollo de las metas de la educación y a inspirar a los otros con una visión de futura del centro.

2. Desarrolla las Personas.

Los líderes eficaces ejercen influencia sobre el desarrolla de los recursos humanos en su centro y lo consideran una prioridad. Las capacidades y motivaciones que los miembros del centro requieren para moverse productivamente en la dirección establecida, están influenciadas por las experiencias que los miembros de la organización tienen con los líderes y lo que perciben en el contexto organizativo.

3. Promueve la transformación Organizativa.

Esta categoría de prácticas de liderazgo, ha surgido según Leithwood, de las evidencias recientes acerca de la naturaleza de las organizaciones que aprenden y de las comunidades profesionales de aprendizaje y su contribución al trabajo de los profesores y el aprendizaje de los alumnos.

4. Gestión del Currículum Escolar.

Los líderes tienen que disponer de información actualizada acerca de las mejores prácticas profesionales y contribuir a desarrollar las condiciones para la mejora de los procesos de enseñanza-aprendizaje del centro, así como de las condiciones para el aprendizaje y desarrolla profesional del profesorado.

Véase en el estudio empírico 4, los indicadores de cada una de las cuatro dimensiones.

\section{Estudios Empíricos}

\section{Estudio Empírico: Liderazgo Situacional}

Durante décadas la teoría de liderazgo situacional fue la más extendida, y aplicada desde la aportación de modelos de diferentes autores (Blanchard \& Jersey, 1975; Fiedler, 1972; Fiedler, 1967; Reddin 1970).

El modelo de liderazgo de Fiedler (1972) funciona del siguiente modo: cada líder debe contestar un instrumento sobre el que se obtiene una puntuación que indica su estilo predominante: Orientado a la Relación u Orientado a la Tarea.

Una alta puntuación en el instrumento Least Preferred Coworker (LPC), el Trabajador menos Preferido, indica una motivación orientada a la relación, y los líderes obtienen su principal satisfacción cuando tienen unas buenas relaciones con los miembros de su equipo y/o profesorado. 
Una puntuación baja en LPC indica una motivación orientada a la tarea del líder. Los líderes obtienen su máxima motivación cuando logran que las cosas se realicen y se lleven a cabo con éxito.

El comportamiento puede variar por parte del líder en función del control, que Fiedler (1972) señala en tres niveles: bajo, medio y alto control. Esto es válido para ambos estilos de liderazgo tanto para el orientado a la relación cómo el orientado a la tarea.

La situación se define en tres variables:

- Relaciones con el líder. Grado en que las personas siguen, son honestos y leales con su líder.

- Estructura de tarea. Grado en el que las tareas están claras para los trabajadores, las pueden llevar a cabo, y son conscientes de las actividades que tienen que desarrollar.

- Posición de poder. Es la suma del poder legítimo, por recompensa y/o coercitivo que tiene un líder por la jerarquía que tiene por su posición en el organigrama institucional.

El modelo de Fiedler se puede ver de forma gráfica en la figura 2.

\section{Resultados de la Investigación de situación en el liderazgo, de Fiedler.}

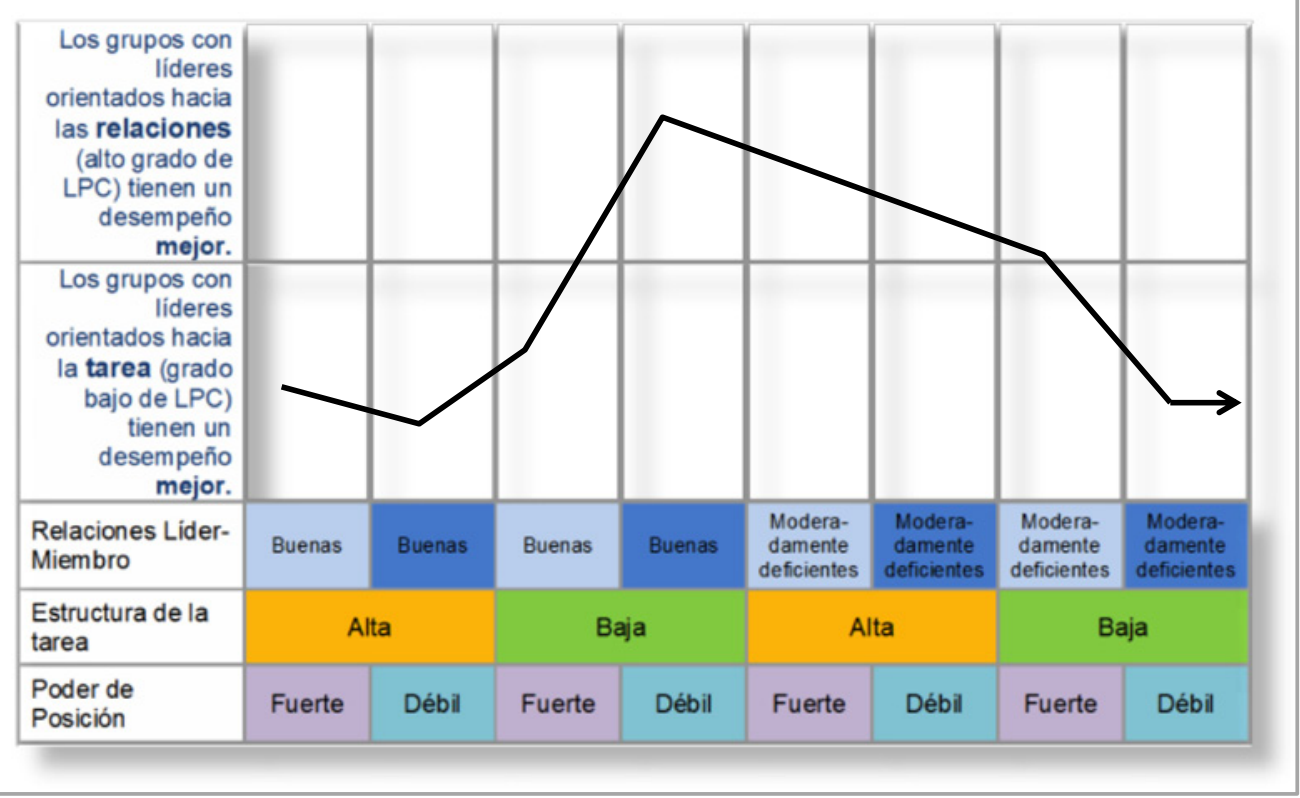

Figura 2. Resultados de la investigación de situación en el liderazgo. Fiedler (1972). 
Durante la Reforma de las Enseñanzas Medias, conocida bajo el acrónimo de REM, tuve la oportunidad de dirigir el proceso de evaluación de la misma, bajo un equipo de profesionales de la Universidad del País Vasco (UPV) y la Universidad de Deusto. En éste extenso estudio, con un seguimiento de más de 3000 estudiantes durante tres años, aplicamos el Modelo de Fiedler para analizar el funcionamiento de la dirección escolar. Elegimos éste modelo denominado enfoque situacional, ya que consideramos que era muy adecuado en un contexto de reforma que se estaba viviendo. Los resultados permitieron conocer la valoración del profesorado de los 47 centros participantes sobre cómo la dirección gestionaba la situación de cambio del modelo educativo y los recursos que lograba o no lograba para promover una situación exitosa. Los resultados obtenidos, también nos permitieron conocer cuáles eran los liderazgos más efectivos en función de la situación distinta que tenían que tener en cuenta. El 78\% de los directores tenía una edad inferior a los 40 años, por tanto, se trataba de directores/as jóvenes en su mayoría.

En nuestra investigación en la REM realizamos cuatro preguntas a los profesores de los 47 centros participantes para analizar la eficacia de la dirección según el profesorado que debían contestar en la siguiente escala: de 1, nada eficaz, a 5 muy eficaz.

La eficacia de la dirección la consideramos en cuatro aspectos que formulamos cómo cuestiones al profesorado de los centros experimentales:

- planificar y diseñar la puesta en práctica (tabla 2).

- la puesta en práctica del Plan experimental de la REM (tabla 3).

- proporcionar al profesorado los recursos necesarios para llevar a cabo el Plan (tabla 4).

- ayudar a resolver sus problemas en su tarea docente (tabla 5).

Tabla 2

Primer Criterio: Eficacia de la dirección en la planificación del plan Reforma

\begin{tabular}{lccccc}
\hline Fuente & GL & Suma Cuadrado & Cuadrados Medios & F & Prob \\
\hline Dentro de los grupos & 1 & 4.5125 & 4.5125 & 13.51 & .0008 \\
Entre los grupos & 36 & 12.022 & .3339 & & \\
TOTAL & 37 & 16,5345 & & & \\
\hline
\end{tabular}

Tabla 3

Segundo Criterio: Eficacia de la dirección en la puesta en práctica del plan Reforma

\begin{tabular}{lccccc}
\hline Fuente & GL & Suma Cuadrado & Cuadrados Medios & F & Prob \\
\hline Dentro de los grupos & 1 & 4.2919 & 4.2919 & 12.62 & .0001 \\
Entre los grupos & 36 & 12.2402 & 0.340 & & \\
TOTAL & 37 & 16.531 & & & \\
\hline
\end{tabular}


Tabla 4

Tercer Criterio: Eficacia de la dirección en proporcionar los recursos necesarios

\begin{tabular}{lccccc}
\hline Fuente & GL & Suma Cuadrado & Cuadrados Medios & F & Prob \\
\hline Dentro de los grupos & 1 & 2.4179 & 2.4179 & 6.9993 & .01 \\
Entre los grupos & 36 & 12.4364 & .3455 & & \\
TOTAL & 37 & 14.8543 & & & \\
\hline
\end{tabular}

Tabla 5

Cuarto Criterio: Eficacia de la dirección en ayudar a resolver problemas en las tareas docentes

\begin{tabular}{lccccc}
\hline Fuente & GL & Suma Cuadrado & Cuadrados Medios & F & Prob \\
\hline Dentro de los grupos & 1 & 2.6796 & 2.4179 & 6.7141 & .01 \\
Entre los grupos & 36 & 14.3675 & .3991 & & \\
TOTAL & 37 & 17.0471 & & & \\
\hline
\end{tabular}

Los cuatro análisis de las cuestiones planteadas dieron como resultado diferencias estadísticamente significativas e importantes entre el liderazgo establecido según el modelo de Fiedler para catalogar como líder eficaz o ineficaz dependiendo del estilo y control de las situaciones establecidas, según el juicio del profesorado (Villa, 1990).

El estilo de dirección es una variable importante y significativa a la hora de plantear un cambio en la enseñanza. En la dirección recae una gran responsabilidad para que las reformas se lleven a cabo. La dirección tiene la responsabilidad de procurar crear las condiciones necesarias, tanto físicas como psicológicas, para que el cambio se produzca y se produzca del modo más efectivo posible. Los cuatro aspectos estudiados en esta investigación son muy similares a los que plantea Day (2013) en su modelo de prácticas de dirección exitosa, aunque éste autor enumera otros factores. El primer aspecto se refiere como rediseño y enriquecimiento del currículum, el segundo aspecto reestructuración de la organización y rediseño de los roles y responsabilidades, el tercer aspecto mejora de las condiciones para la enseñanza y el aprendizaje, y finalmente, el cuarto aspecto fomento de la calidad de la enseñanza-aprendizaje.

\section{Estudio empírico: Liderazgo Transformacional}

Bajo éste enfoque de liderazgo transformacional, utilizamos el modelo de Bernard Bass al que le habíamos invitado a trabajar con nosotros y se adaptó su cuestionario al castellano. Realizamos dos investigaciones, la primera en el ámbito empresarial y la segunda en el ámbito educativo. 


\section{A) Liderazgo Transformacional en el ámbito empresarial.}

La primera vez que se aplicó el modelo de Bass en una investigación en España se llevó a cabo en el ICE de la Universidad de Deusto bajo la dirección de Roberto Pascual, en una muestra de empresas (Pascual, Villa \& Sánchez, 1990).

El estudio en el ámbito empresarial se realizó en una muestra proporcionada a las tres provincias vascas (Álava, Vizcaya \& Guipúzcoa) con una participación de 112 empresas, 96 directivos y 265 empleados.

Se correlacionaron los cuatro factores del liderazgo Transformacional, los dos de Liderazgo Transaccional y el de No liderazgo (Dejar Hacer) con tres variables: Eficacia organizacional, satisfacción y el esfuerzo extra percibido por los empleados.

A continuación, presentamos la tabla 6.

Tabla 6

Correlaciones de los Factores de Liderazgo con las variables de Eficacia organizacional, satisfacción y esfuerzo extra

\begin{tabular}{lccc}
\hline Liderazgo Transformacional & Eficacia & Satisfacción & Esfuerzo Extra \\
\hline Influencia Idealizada & $.76^{* *}$ & $.83^{* *}$ & $.85^{* *}$ \\
Motivación Inspiracional & $.43^{* *}$ & $.53^{* *}$ & $.42^{* *}$ \\
Consideración Individual & $.45^{* *}$ & $.50^{* *}$ & $.78^{* *}$ \\
Estimulación intelectual & $.64^{* *}$ & $.61^{* *}$ & $.53^{* *}$ \\
Liderazgo Transaccional & & & $.46^{* *}$ \\
Dirección contingencial & $.26^{* *}$ & $.30^{* *}$ & $.17^{* *}$ \\
Dirección por Excepción & $.23^{* *}$ & $.26^{* *}$ & \\
No Liderazgo & & & $-.34^{* *}$ \\
Dejar Hacer & $-.54^{* *}$ & $-.42^{* *}$ & \\
\hline
\end{tabular}

Nota: ${ }^{* *}$ Indica que el resultado de la $\mathrm{r}$ es significativo al $99 \%$.

Como puede advertirse, todos los factores que configuran el liderazgo transformacional muestran una asociación más alta que cualquiera de los factores transaccionales, y el estilo de liderazgo "laissez faire" correlaciona negativamente con las tres variables estudiadas: eficacia, satisfacción y esfuerzo extra.

\section{B) El Liderazgo Transformacional en Educación}

Pascual, Villa y Auzmendi (1993) llevaron a cabo un estudio empírico en las Comunidades de Castilla-León con una población de 1261 centros educativos y en el País Vasco con un total de 716 centros. La muestra sobre la que se llevó a cabo el estudio se expone en la tabla 7. 
Tabla 7

Muestra del estudio de Pascual et al. (1993).

\begin{tabular}{lccc}
\hline Comunidad Autónoma & $\begin{array}{c}\text { Centro educativo } \\
\text { público }\end{array}$ & $\begin{array}{c}\text { Centro educativo } \\
\text { privado }\end{array}$ & Total \\
\hline Castilla y León & 17 & 11 & 28 \\
Andalucía & 13 & 15 & 28 \\
TOTAL & 30 & 26 & 56 \\
\hline
\end{tabular}

Se realizó un análisis factorial de componentes principales con rotación varimax emergiendo los siguientes factores:

- El estilo transformacional: Carisma Personalizador, Tolerancia Psicológica (uso del sentido del humor), Inspiración, y Liderazgo hacia arriba.

- El estilo transaccional: dos factores Liderazgo por Contingencia y Liderazgo por Excepción.

- El no liderazgo o Laissez-faire.

Se analizó la asociación del estilo de liderazgo con cuatro variables, que se exponen en la tabla 8.

Tabla 8

Correlaciones entre las dimensiones de Liderazgo y las cuatro variables estudiadas

\begin{tabular}{lcccc}
\hline Liderazgo Transformacional & Eficacia & $\begin{array}{c}\text { Satisfacción del } \\
\text { profesorado }\end{array}$ & $\begin{array}{c}\text { Satisfacción } \\
\text { de la dirección }\end{array}$ & $\begin{array}{c}\text { Esfuerzo } \\
\text { extra del } \\
\text { profesorado }\end{array}$ \\
\hline Carisma Personalizador & $.76^{* *}$ & $.53^{* *}$ & $.85^{* *}$ & $.85^{* *}$ \\
Tolerancia Psicológica & $.43^{* *}$ & $.32^{* *}$ & $.53^{* *}$ & $.42^{* *}$ \\
Inspiración & $.45^{* *}$ & $.33^{* *}$ & $.50^{* *}$ & $.78^{* *}$ \\
Liderazgo hacia Arriba & $.64^{* *}$ & $.47^{* *}$ & $.61^{* *}$ & $.53^{* *}$ \\
Liderazgo Transaccional & & & & $.30^{* *}$ \\
Liderazgo por Contingencia & $.26^{* *}$ & $.16^{* *}$ & $.26^{* *}$ & $.16^{* *}$ \\
Liderazgo por Excepción & $.23^{* *}$ & $.20^{* *}$ & & \\
No Liderazgo & & & $-52^{* *}$ & $.34^{* *}$ \\
Dejar Hacer (Laissez Faire) & $-.54^{* *}$ & $-.32^{* *}$ & $-52^{* *}$ \\
\hline
\end{tabular}

Nota: ${ }^{* *}$ Indica que el resultado de la $\mathrm{r}$ es significativo al $99 \%$ 
La conclusión principal que queda claramente demostrado es que el liderazgo transformacional tiene un impacto en el centro educativo significativamente mayor que cualquier otro estilo de liderazgo, y esto sucede con cualquier de los factores que configuran el estilo del liderazgo transformacional.

El modelo de liderazgo transformacional ha sido estudiado en múltiples ocasiones: Filella (1989), Muñoz (2004), Murillo (2006), Bennetts (2007). Subrayamos la obra de Oscar Maureira (2004), por su enfoque causal que es muy interesante y con escasos estudios.

Un estudio realizado por Sun y Leithwood (2014) basado en una revisión de la investigación de catorce años que incluye un meta-análisis sobre el liderazgo transformacional de la escuela (LTE) presenta unas interesantes conclusiones:

- La mayoría de los estudios sobre los efectos de LTE en el logro de los estudiantes, hasta la fecha, se han basado en concepciones estrechas del liderazgo transformacional, concepciones que no reconocen el contexto organizacional de los líderes. (Según los autores, es necesario ampliar el liderazgo transformacional más el liderazgo instructivo, lo que denominan un liderazgo "integral).

- Algunas prácticas de LTE hacen contribuciones mucho mayores a los logros de los estudiantes que otras. (Subrayan los autores que incluso los modelos estrechamente concebidos han demostrado efectos pequeños pero positivos).

\section{Prácticas Eficaces según el modelo de Kenneth Leithwood}

El modelo comprensivo de Leithwood y colaboradores (Leithwood et al., 2006; Leithwood \& Riehl, 2005), describe cuatro categorías generales o dimensiones de liderazgo exitosas que a su vez agrupan una serie de prácticas de liderazgo:

1. Establecer direcciones:

1.1. Identifica y articula una visión.

1.2. Promueve la aceptación de las metas del grupo.

1.3. Expectativas de alto rendimiento.

2. Desarrollar las personas:

2.1. Proporciona consideración individualizada.

2.2. Proporciona estimulación intelectual.

2.3. Se comporta con integridad personal y profesional.

3. Promueve la transformación organizativa:

3.1. Facilita el desarrollo de una cultura de colaboración.

3.2. Transforma las estructuras para promover la colaboración.

3.3. Desarrolla relaciones positivas con las familias y la comunidad.

4. Gestión del currículo escolar:

4.1. Planifica y supervisa la enseñanza.

4.2. Proporciona apoyo instructivo.

4.3. Hace seguimiento del progreso del centro.

4.4. Favorece un clima de trabajo docente adecuado para el logro de las prioridades. 
Las prácticas de liderazgo constituyen la definición operativa de cada una de las cuatro dimensiones del modelo y definen las conductas de los/las líderes tal y como son percibidas por sus colaboradores y el conjunto del profesorado. Por tanto, la evaluación del ejercicio del liderazgo desde este modelo de Liderazgo Transformacional Educacional nos permite obtener un perfil comprensivo de la práctica del liderazgo y la gestión en contextos educativos.

El estudio fue realizado en 19 centros educativos de Guipúzcoa (Villa \& Gorriño, 2009), procesándose 822 cuestionarios válidos. Del total de centros, trece son privados concertados y seis son públicos. En cuanto al nivel educativo que imparten, de los trece centros concertados, cuatro imparten ESO exclusivamente, ocho centros impartían ESO y Bachillerato y uno imparte ESO, Bachillerato y Ciclos Formativos Medio y Superior. De los seis centros públicos, uno impartía en ESO y Bachillerato y los cinco restantes con Ciclos Formativos Medio y Superior.

El instrumento utilizado fue un cuestionario traducido y adaptado de Leithwood. Puede verse una versión preparada para la autorrevisión de estas prácticas en Villa y Gorriño (2009). El cuestionario recoge las cuatro dimensiones descubiertas según la investigación de Leithwood et al. (2006) y replicadas en diversos estudios en diferentes países. Se recogen algunos resultados en la tabla 9.

Tabla 9

Resultados de la escala aplicada de Leithwood por Villa y Gorriño (2009).

\section{Escala Total}

$(n=852)$

Promueve la Transformación Organizativa
Establece direcciones

Desarrolla a las personas

Gestión del currículo escolar
Consistencia interna de las escalas (Alpha de Cronbach)

Entre los resultados principales es destacable que, al respecto de la primera hipótesis nula, no existe diferencia significativa en los niveles de liderazgo de los directivos de los centros participantes. Para comprobar esta hipótesis se seleccionó al 20\% de los centros con mayor puntuación y otro $20 \%$ constituido por los centros con menor puntuación. Así se ha procedido a contrastar las puntuaciones de los grupos 1 y 4 , mediante la prueba estadística "t de Student" para la igualdad de medias de muestras independientes. Los resultados se sintetizan en la tabla 10. 
Tabla 10

Prueba t de Student para la igualdad de medias.

\begin{tabular}{lccccccc}
\hline Dimensión & $\mathrm{t}$ & $\mathrm{gl}$ & $\begin{array}{c}\text { Sig. } \\
\text { (bilateral) }\end{array}$ & $\begin{array}{c}\text { Diferencias } \\
\text { de medias }\end{array}$ & $\begin{array}{c}\text { Error típ. } \\
\text { de la dif. }\end{array}$ & $\begin{array}{c}95 \% \text { Inter } \\
\text { confianza } \\
\text { inferior }\end{array}$ & $\begin{array}{c}95 \% \text { Inter } \\
\text { confianza } \\
\text { superior }\end{array}$ \\
\hline Establece Direcciones & 6.584 & 333 & .000 & .40735 & .06187 & .28565 & .52906 \\
Desarrolla Personas & 6.943 & 322.196 & .000 & .46001 & .06625 & .32967 & .59035 \\
Gestión del Currículo & 6.500 & 320.468 & .000 & .38781 & .05966 & .27043 & .50519 \\
$\begin{array}{l}\text { Promueve Transfor- } \\
\text { mación }\end{array}$ & 7.686 & 310.431 & .000 & .46877 & .06099 & .34877 & .58877 \\
\hline
\end{tabular}

Como puede observarse, se evidencian diferencias significativas entre el grupo 1 y el grupo 4, al encontrarse que los valores del nivel de significación (indicado como "Sig. Bilateral" en la tabla y que corresponde a la probabilidad de cometer un error de tipo I, esto es rechazar la hipótesis nula, siendo ésta verdadera; es también conocido como margen de error, que por convención general se establece en un $5 \%$ o 0.05 .) son inferiores a 0.001 en todas las dimensiones del liderazgo analizadas, por tanto, se debe rechazar la hipótesis nula y aceptar la hipótesis alterna que indica que existen diferencias significativas en los niveles de liderazgo de los directivos de los centros participantes (la tendencia se registra de la misma manera en el total general) . A este respecto, si se observa la columna "diferencia de medias", se nota que los valores son positivos, lo que estaría indicando que los niveles más altos de liderazgo se dan en los centros del grupo 1 y que éstos superan a sus pares del grupo 4 en todas las dimensiones.

En éste estudio se llevó a cabo el examen del fenómeno denominado por Bass efecto dominó o efecto cascada, por primera vez en España. Si bien es cierto que la muestra era de tan solo 17 centros educativos. En cada uno de los centros se establecieron tres niveles de liderazgo, cada uno con sus correspondientes evaluaciones. En el primer nivel, se evaluó la figura del Director General (en su caso), Director, Director Adjunto y/o Subdirector. En el segundo nivel, Directores pedagógicos, Jefes de estudios y Coordinadores o Responsables de Etapa. Finalmente, el tercer nivel lo constituyen los Jefes de departamento, Jefe de proceso o cargos similares. El efecto dominó significa que cuando el equipo directivo manifiesta un estilo de liderazgo transformacional lo traspasa a su equipo y éste al siguiente nivel, si este Segundo equipo manifiesta un claro liderazgo trasnformacional lo transciende al siguiente nivel. En nuestro estudio, a pesar de tener una muestra reducida pudimos comprobar que los centros que sus tres niveles de liderazgo tenían un estilo transformacional obtuvieron una diferencia significativa en todas las variables comparándolos con sus respectivos equipos homólogos.

Esto significa que el liderazgo transformacional no es un liderazgo personal, sino que se comparte, que ayuda a todos los miembros, que se transmite el entusiasmo y 
se logra una identificación con la visión. Se abre una línea interesante de investigación para conocer con mayor profundidad cómo se transciende en los equipos y personas de cada nivel directivo.

\section{Estudio empírico: Liderazgo Emocional}

Hemos unido dos instrumentos que nos han permitido elaborar un modelo operativo que estamos aplicando en la formación de directivos, y que estamos comprobando que ofrece muy buenos resultados para entender y explicar el comportamiento emocional de los líderes educativos. Estos dos instrumentos son el Wleiss y el de Sterret (2002), como se muestra en la figura 3.

\section{INTELIGENCIA EMOCIONAL}

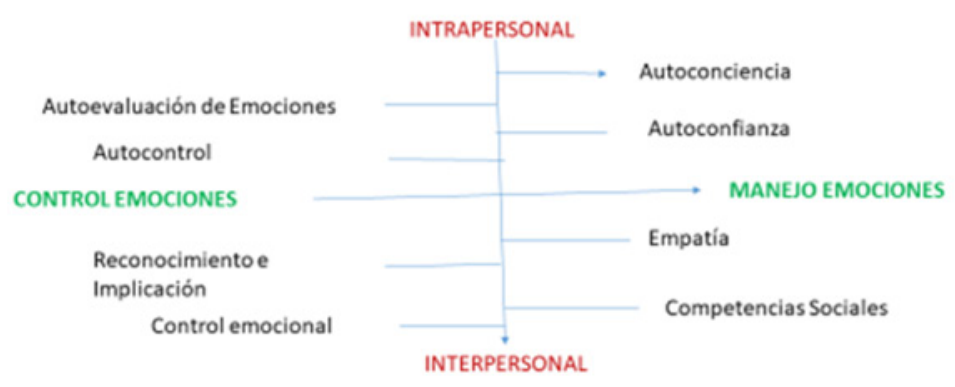

Figura 3. Inteligencia Emocional. Modelo operativo uniendo instrumentos Wleiss y Sterret.

Este modelo compuesto por los cuatro factores del WLEIS configuran los dos ejes del plano. El eje vertical está determinado por el factor Intrapersonal-factor Interpersonal, y el eje horizontal por el Manejo de las Emociones-Control Emocional. Estos dos ejes, a nuestro entender configuran bien el marco emocional, y las dimensiones de Sterret (2002) aportan las emociones más concretas y específicas lo que favorece un conocimiento profundo de las emociones. En el área Intrapersonal, hay dos emociones clave la autoconciencia y la autoconfianza. En el Manejo de emociones, destacamos dos aspectos: la empatía (cómo la capacidad de ponerse en el lugar del otro), y las competencias sociales (las habilidades para relacionarse e interactuar con los demás). En el control de las emociones interpersonales (señalamos el reconocimiento e implicación y el control emocional). Finalmente, en el control de las emociones en el área intrapersonal, se sitúan la autoevaluación (la propia capacidad de reconocer y valorar tu comportamiento emocional, y la habilidad de autocontrol en determinadas situaciones).

Los dos instrumentos que complementariamente se usan en la investigación, fueron validados en dos estudios. El primero el denominado WLEIS, (cuyos autores son Wong y Law, 2002), fue traducido y adaptado al contexto español (Urquijo et al., 2015), los cuatro factores hallados obtuvieron una alta consistencia interna, como se indica a continuación. 
Percepción Intrapersonal $(\alpha=.86)$, entendida cómo la capacidad del individuo para entender sus emociones profundas y ser capaz de expresar estas emociones de forma natural. Percepción Interpersonal $(\alpha=.85)$, entendida cómo capacidad de evaluación y reconocimiento de las emociones en los demás (valoración emocional de los demás. Asimilación Emocional $(\alpha=.79)$ entendida cómo capacidad de las personas para regular sus emociones, lo que permitirá una recuperación más rápida de trastornos psicológicos.

Y finalmente, la Regulación Emocional ( $\alpha=.82)$, definida cómo capacidad de las personas para hacer uso de sus emociones, dirigiéndolos hacia una constructiva actividad y desempeño personal.

El cuestionario de Sterret (2002) proporciona información en los siguientes ámbitos:

- Ámbito de las capacidades propias de la dimensión del Yo tales como la autoconciencia, la autoconfianza y el autocontrol.

- Ámbito de las capacidades propias de la dimensión social cómo: la empatía, la motivación y las competencias sociales.

Los resultados obtenidos, aunque son todavía muy provisionales por provenir de unas muestras reducidas, son sin embargo muy prometedores.

\section{Discusión y conclusiones}

Durante este desarrollo de estas casi cuatro décadas podemos constatar algunas consideraciones claras provenientes de la abundante revisión bibliográfica de estudios e investigaciones sobre el tema:

- Cambio de tendencia de la consideración de una tarea individual hacia una tarea colegiada y compartida.

- Baja representatividad en la dirección de la mujer a pesar de estar en un ámbito muy feminizado.

- Una consideración desde la investigación de la necesidad de una profesionalización de la dirección en España, aunque parcialmente se han ido realizando modificaciones como mayor duración en el cargo, una formación directiva básica.

- La falta de asunción de la responsabilidad de la dirección en algunos aspectos de la gestión directiva lo que provoca una debilidad de la dirección en los centros públicos.

Renace con fuerza el liderazgo pedagógico como una tarea importante de la dirección, para promover, asegurar y evaluar el aprendizaje de los estudiantes. Los estudios empíricos refuerzan la conclusión que cuando existe un liderazgo en el centro independientemente del modelo utilizado tiene un impacto positivo, lo que no significa que no existan modelos más eficaces que otros, que confirma lo que ha señalado la OCDE (2014): no hay un buen funcionamiento de un centro sin liderazgo directivo.

El modelo de prácticas eficaces de Leithwood y colaboradores, aplicado en diferentes países y contextos muy diversos, emerge como un modelo a promocionar por los 
buenos resultados que se logran con el mismo. El liderazgo emocional es un aspecto clave en el liderazgo educativo y requiere una mayor atención en la formación de los equipos directivos.

\section{Referencias}

Álvarez, M. (2010). Liderazgo compartido. Buenas prácticas de dirección escolar. Madrid: Wolters Kluwer.

Bass, B.M. (1981). Stodgill's Handbook of Leadership. New York: Free Press.

Bass, B.M. (1985). Leadership and performance beyond expectations. New Cork: Free Press. Bayley, T. (2000). Desarrollo de la Dirección de Centros y el Papel de la Formación en ejercicio: Avances recientes en Inglaterra y Gales. En R. Pascual y A. Villa (Coords.), La Dirección factor clave de la Calidad Educativa. Bilbao: ICE de la Universidad de Deusto.

Bennetts, S. (2007). El liderazgo transformacional y la evaluación de programas académicos universitarios en México. Educar, 40, 163-189.

Blanchard, K. (2007). Liderazgo de máximo nivel. Barcelona: Granica.

Bolívar, A. (2012). Políticas actuales de mejora y Liderazgo Educativo. Málaga: Aljibe.

Boyatzis, R. E., McKee, A., \& Johnston, F. (2008). Líder emocional: Manual de uso. Barcelona: Deusto.

Cameron, K.S., Dutton, J.E., \& Quinn, R.E. (Eds.) (2003). Positive Organizational Scholarship. San Francisco: Berret-Koehler Publishers.

Castiñeira, A., \& Lozano, J.M. (2012). El poliedro del liderazgo. Una aproximación a la problemática de los valores en el liderazgo. Barcelona: Libros de Cabecera.

Day, C. (2013). Prácticas exitosas de liderazgo educativo desde una perspectiva comparada. En A. Villa (Ed). Liderazgo pedagógico en los centros educativos. Bilbao: Mensajero.

Earley, P., \& Greany, T. (Eds.) (2017). School Leadership and Education System Reform. London: Bloomsbury.

Earley, P., \& Greany, T. (2019). The Context and Challenges of Contemporary School Leadership. En A. Berkeley y E. Jackson (Eds.), Keeping Your Head: finding and sustaining balance, depth and resilience in school leadership. London: Karnac Press.

Estruch, J. (2002a). Dirección profesional y calidad educativa. Barcelona: Praxis.

Estruch, J. (2002b). Hacia la profesionalización de la dirección de centros escolares. Revista de Educación, 329. Recuperado de http://www.educacionyfp.gob.es/dctm/revista-deeducacion/articulosre329/re3290511165.pdf?documentId=0901e72b812593ae

Fiedler, F. (1967). A Theory of Leadership Effectiveness. New York: McGraw Hill.

Fiedler, F. (1972). How do you Make Leaders More Effective. New Answers to an Old Puzzle.

Filella, J. (1989). La práctica del liderazgo transformacional. Boletín de Estudios Económicos, 44(136), 73-88.

Fisher, R., \& Sharp, A. (1999). El liderazgo lateral. Madrid: Gestión 2000.

Gairín, J., \& Villa, A. (1996). Los equipos directivos. Análisis y reflexión a partir de una investigación empírica. En Villa, A. (Ed.) Dirección Participativa y evaluación de centros. Bilbao: Mensajero.

García-Olalla, A. (1998). Análisis del funcionamiento de los equipos directivos de centros educativos en el contexto del Estado Español (Tesis doctoral). Bilbao: Universidad de Deusto. 
García-Olalla, A., \& Poblete, M. (2003). El desarrollo profesional en la dirección de centros educativos, hacia la construcción de un modelo basado en competencias. En Congreso Internacional: Humanismo para el siglo XXI. Bilbao: Universidad de Deusto.

García-Olalla, A., Poblete, M. y Villa, A. (2006). La función directiva: un problema sin resolver. Tres décadas de formación, investigación y acción. XXI. Revista de Educación, 8, 13-34.

Goleman, D. (1996). Liderazgo. El poder de la inteligencia emocional. Barcelona: B.S.A.

Goleman, D. (1998). La práctica de la inteligencia emocional. Barcelona: Kairós.

Goleman, D., \& Cherniss, C. (2013). Inteligencia emocional en el trabajo. Barcelona: Kairós.

Goleman, D., Boyatzis, R., \& McKee, A. (2002). El líder resonante crea más. Barcelona: Plaza y Janés.

Greenleaf, R.K. (1977). Servant Leadership. New York: Paulist Press.

Gronn, P. (2002). Distributed leadership. In K.A. Leithwood and Halligenger (Eds.). Second International Handbook of Educational Leadership and Administration. Dpordrecht: Kluwer.

Heifetz R. (1994). Leadership Without Easy Answers. Cambridge: Harvard University Press.

Lewin, K., Lippitt, R., \& White, R.K. (1939). Patterns of aggressive behavior in experimentally created social climates. Journal of Social. Psychology, 10, 271-279.

Lowney, C. (2004). El liderazgo al estilo de los jesuitas. Barcelona: Granica.

MacBeath, J. (2011). Liderazgo el aprendizaje dentro de la escuela. Santiago de Chile: Centro de Innovación en Educación de Fundación Chile.

Marzano, R.J., Waters, T., \& McNulty, B.A. (2005). School Leadership what works: From Research to Results. Alexandria, VA: Association for Supervision and Curriculum Development (ASCD).

Maureira, O. (2004). Liderazgo y eficacia escolar: hacia un modelo causal. Santiago de Chile: Ediciones UCSH.

McInermy, P. (2003). Moving into dangerous territory? Educational leadership in a devolving education system. International Journal Leadership in Education, 6(1), 57-72.

Mulford, B. (2006). Liderazgo para mejorar la calidad de la educación secundaria. Profesorado. Revista de Currículum y Formación del Profesorado, 10(1), 1-22.

Muñoz, D. (2004). Mujeres emprendedoras: liderazgo transformacional y género. Iniciativa Emprendedora y Empresa Familiar, 44, 126.

Murillo, J. (2006). Una dirección escolar para el cambio: del liderazgo transformacional al liderazgo distribuido. Revista Electrónica Iberoamericana sobre Calidad, Eficacia y Cambio en Educación, 4(4), 11-14.

Murphy, J. (1988). Methodological, measurement, and conceptual problems in the study of instructional leadership. Educational Evaluation and Policy Analysis, 10(2), 117-139.

OCDE (2014). La reserche et l'innovation dans l'enseigment. Environnements pédagogiques et practices novatrices. París: OCDE.

Pascual, R. (1987). Liderazgo y participación: Mitos y Realidades. Bilbao: Universidad de Deusto.

Pascual, R., \& Villa, A. (1991). La dirección factor clave de la calidad educativa. Bilbao: ICE de la Universidad de Deusto.

Pascual, R., Villa, A., \& Auzmendi, E. (1993). El liderazgo Transformacional en los centros docentes. Bilbao: Mensajero. 
Pascual, R., Villa, A., \& Sánchez, M. (1990). El liderazgo en la empresa vasca. Bilbao: ICE de la Universidad de Deusto.

Pillans, G. (2015). Leadership Development: Is it Fit for Purpose? London: Corporate Research Forum.

Reddin, W. (1970). Managerial Effectiveness. New York: McGraw Hill.

Sashkin, M. (1998). Líder Visionario. Madrid: Centro de Estudios Ramón Areces.

Segarra, O. (2018). La evolución del liderazgo peregrine. Barcelona: Libros de Cabecera.

Sheppard, B. (1996). Exploring the Transformational Nature of Instructional Leadership. The Alberta Journal of Educational Research, 42(4), 325-344.

Simkins, T. (2012). Understanding school leadership and management development in England: retrospect and prospect. Educational Management, Leadership and Administration, 40(5), 621-640. doi: http://dx.doi.org/10.1177/1741143212451172

Spillane, J., Halverson, R. and Diamond, J. (2001). Investigating school leadership practice: a distributive perspective. Educational Researcher, 30(3), 23-28.

Sterret, E. (2002). Guía del directivo para la inteligencia emocional de la gestión del liderazgo. Madrid: Centro de Estudios Ramón Areces.

Stodgill, R.M. (1974). Handbook of Leadership. New York: Free Press

Urquijo, I., Extremera, N., \& Villa, A. (2015). Emotional intelligence, life satisfaction and psychological well-being in graduates: the mediating effect of perceived stress. Applied research in quality of life, 11(4), 1241-1252. doi: http://dx.doi.org/10.1007/ s11482-015-9432-9.

Villa, A. (1990). Reforma de las Enseñanzas Medias. Evaluación del primer ciclo del plan experimental en la C.A.V. Vitoria: Servicio Central de Publicaciones del Gobierno Vasco.

Villa, A. (Ed.) (2000). Liderazgo y Organizaciones que aprenden. Bilbao: ICE de la Universidad de Deusto.

Villa, A. (Ed.) (2004). Dirección para la Innovación: apertura de los centros a la Sociedad del Conocimiento. Bilbao: ICE de la Universidad de Deusto.

Villa, A. (Ed.) (2008). Innovación y cambio en las organizaciones educativas. Bilbao: ICE de la Universidad de Deusto.

Villa, A. (Ed.) (2013). Liderazgo Pedagógico en los centros educativos. Competencias equipos directivos, profesorado y orientadores. Bilbao: ICE de la Universidad de Deusto.

Villa, A. (2009). El perfil del liderazgo en los centros educativos de enseñanzas medias en Guipúzcoa. San Sebastián: Informe de investigación para la Diputación Foral de Guipúzcoa.

Villa, A., \& García-Olalla, A. (2003). El procedimiento e acceso a la dirección. Reflexiones y propuestas para el debate. Organización y gestión Educativa, 2, 4-14.

Villa, A., \& Gorriño, M. (2009). El liderazgo en los centros de Educación Secundaria. En L.M. Villar, Creación de la Excelencia en Educación Secundaria (pp. 61-90). Madrid: Pearson.

Villa, A., Auzmendi, E., \& Villardón, L. (1996). Los equipos directivos ante el uso de la evaluación. Creencias, actitudes y conductas directivas. Bilbao: Mensajero.

Wong, C.S., \& Law, K.S. (2002). The effects of leader and follower emotional intelligence on performance and attitude: An exploratory study. Leadership quarterly, 13, 243-274. doi: https://doi.org/10.1016/S1048-9843(02)00099-1 
Zepke, N. (2007). Leadership, power and activity systems in a higher education context: will distributive leadership serve in an accountability driven world? International Journal of Leadership in Education, 10(3), 301-314. doi: https://doi. org/10.1080/13603120601181514

Fecha de recepción: 4 de marzo de 2019.

Fecha de revisión: 4 de marzo de 2019.

Fecha de aceptación: 28 de marzo de 2019. 